\title{
Management Theories on Leadership in Pre-University Education
}

\author{
Veronica-Claudia Istratie (Achim) \\ veronique claudia@yahoo.com \\ Ruxandra Georgescu \\ gruxandra11@yahoo.ro \\ Gabriela Daniela Bulacu \\ Valahia University of Targoviste, Romania
}

\begin{abstract}
The concept of leadership took different forms and connotations in contemporary society. Leadership is no longer just about giving orders, leadership has become a form of leadership. In pre-university education, there is a need for leadership on all levels. This article aims to provide convincing information about the concept of leadership in pre-university education.
\end{abstract}

Keywords: preuniversity education, leader.

\section{Introduction}

The inability of researchers who have studied leadership until the 1970s in their attempt to find a direct dependence between leadership, satisfaction, and productivity by defining a universal leadership style effectively constituted an argument to take into account the fact that, in all cases, one or more additional factors act, which has led researchers to develop new approaches to the leadership study. ${ }^{9}$

Research on leadership issues has highlighted two major variables - behavioral dynamics and situational status - according to which the main leadership groups have been identified.

Dynamics of behavior refers to how the leader is viewed and analyzed: static (leadership qualities analysis) or dynamics (analysis of human behavioral patterns). In the first case, leadership is treated mainly in terms of qualities, relatively stable and permanent of the character of the individual, that is, it is believed that the leader would have innate characteristics that are necessary for him to be effective. Opposite to this concept, the behavioral approach is based on the conclusions drawn from the observations made on models of human behavior, that is, on the actions of the leader and not on his innate qualities. The second variable is related to the level or degree of situationality, as the basis of the analysis, within a particular approach in the leadership study

At one pole are the approaches that lead to the idea of universality, at the other pole situation is considered decisive for an efficient leadership. ${ }^{10}$

Management research has evolved rapidly, moving from one paradigm to another in terms of leadership and leadership. The main theoretical orientations in this field are as follows:

1) The theory of the providential man (of the "great man"), which emerged at the end of the nineteenth century and the beginning of the twentieth century, is based on the idea that the leader has inherently a series of characteristics and qualities that give him status. The qualities of the leader are seen as inexplicable, which is why such individuals are regarded as heroes. Even though this orientation is no longer in the mood, it is overcome, it still has quite a few followers, who constantly offer examples of such contemporary leaders

2) The theory of traits (the theory of traces) is also one that involves a multitude of discussions today. In the case of this terror, it does not interest who the great people who lead us, but what are the traits they possess. Therefore, this paradigm focuses on the idealization of the features of

9 P. Vagu și I. Stegăroiu, op.cit., p. 191

${ }^{10}$ P. Vagu și I. Stegăroiu, Motivarea in munca : de la teorie la practica p.129 
a leader. However, this is a controversial issue, because many requests that have tried to identify common features of the leaders have not found significant correlations between certain affairs and leadership. This is all the more so since man is an extremely complex system and still incomprehensible in many respects, so it is not advisable to dare to break it down into parts to decipher it.

3) The theory of power and influence centers on the influence and power of leaders. This paradigm starts from the idea that all roads lead to the leader, denying any merit or role of employees or organizational culture. Though at present, this theory is considered to be overcome, in many of the Romanian organizations it is still valid, especially in companies where the leadership of this lip is combined with a mafia-type, in which the leader is only apparently the end of the road.

4) Behavioral theory still has supremacy in many areas. This theory starts from what the leaders actually do, but it does not focus on their features, characteristics. Among the proponents of this theory are Blake, Mouton (the creators of Management Grid) and Rensis Likert. The leader is seen as having a leader-specific behavior, this behavior of the individual changing from the time of his investment in this function. This theory promotes the selection of leaders by behavioral criteria. However, specialized literature has often drawn attention to the risk of such an approach, but people tend to intentionally alter behaviors in test-evaluation situations, which can seriously dispel the results and, therefore, selection. Also, attention has been drawn to the fact that most leaders change their behavior once they become leaders. For this reason, it has been recommended in many companies to take the probation.

5) Situational theory, believes that leadership is more specific to a particular situation than a particular form of expression of the leader's personality. This paradigm starts from the idea, quite plausible, that different circumstances require different forms of leadership. The main supporters of this theory are Kenneth Blanchard and Paul Herse, whose Book - Situational Leadership Theory - remains a true situational manifest. ${ }^{11}$

6) Contingency theory, continues situational theory, with the main purpose of selecting the most appropriate and indicated situational variables, which are suitable for a certain correctly adapted driving style. This theory starts from the idea that it is easier to find a leader to climb a situation than to adapt a particular leader to different situations. The main followers of this theory are Fielder and Vroom-Yetton-Jaro, whose models have contributed significantly to the development of this paradigm.

7) Transactional theory, consider the leader-subaltern relationship. This theory investigates the mutual benefit of the parties, resulting from a relationship that is based on mutual benefits. In this context, the leader offers clear things (such as, for example, rewards or resources) and demands instead recognition of his authority from the subordinates. It is emphasized in this way the role of motivation (and in particular external motivation) in the work of organizations.

8) The theory of attributions, is the theory that raises subordinates on a new stage of importance within organizations. It focuses on the factors behind the personalized managerial duties for each of the employees, as well as on the way the tasks are performed (not on the personal characteristics of the employees).

${ }^{11}$ C. Carnall, Managing Change in Organizations, London, Prentice Hall International Ltd., 1995, p59 
9) Information theory takes into account the intrinsic motivation of employees (for the sake of transactional theory, which focuses on their extrinsic motivation). Within this paradigm, a great emphasis is placed on employee obligations, not on their obedience to leaders. Therefore, transformational lire is innovative and proactive, it is a model that is very close to the ideal leadership model.

Within the educational units, both in Romania and in the world, there is an increasing emphasis on a transformational leader style. Transformation within educational establishments occurs when a leader empowers members of the educational community to improve and adapt from within. A transformational leader simply does not run a particular school, he keeps it afloat, trying to do the best for an authentic collaboration between the members of the school organization and the stakeholders.

\section{Conclusions}

Transformational Leadership promotes the autonomy of all educational actors, empowering them to solve problems and innovate without asking permission and trusting their own expertise. Unfortunately, in some traditional schools, emphasis is still placed on the hierarchical models of leadership, where the decision-making process is carried out vertically, from top to bottom, which has a negative effect on the motivation of teachers, who feel their voice is not matter. Instead, in a transformational school transformational leadership flourishes, and individuals are convinced that their voice counts, which raises the level of collective effectiveness, thus enhancing the pupils' school performance.

Transformational leaders are captains who trust their crew to help design and improve their work in the school. They create a culture of innovation and motivate teachers and students to progress continually, aiming to create the best learning environment for students. Such leaders are aware that transformation is not a unique performance - that they must orient their goals not to appear as singular heroes but as members of a unified effort to transform schools through fearless leadership.

All these practical research challenges underline the interdependence of leadership adopted by executives and teachers. Therefore, as a conclusion, it must be stressed that a new concept, the integrated school leadership, which highlights the synergetic power of the school leader and which is shared by individuals throughout the educational process, needs to be emphasized.

\section{Bibliography}

1. C. Carnall, Managing Change in Organizations, London, Prentice Hall International Ltd., 1995

2. P. Vagu and I. Stegariu, Motivation in Work: From Theory to Practice, 2007 